\title{
ABSCESOS HEPATICOS Y PERITONITIS SECUNDARIA A ASCARIDIOSIS
}

\author{
Por el Dt. FLORENCIO BAEZA DONOSO \\ Hosp. M. Arriatán. Cátedras di Pediatria de los Profs. A. Baeza Goñi y Julio Meneghello R.
}

La ascaridiosis es una entoparasitosis frecuente en la infancia. Neghme' la encuentra en el $46.14 \%$ de niños cuya edad fluctuaba entre 1 a 6 años. Los síntomas atribuídos a la infestación por áscaris son múltiples y se los puede agrupar en generales: palidez, desnutrición y variabilidad del apetito; nerviosos tales como los terrores nocturnos, prurito nasal y síntomas digestivos, describiéndose entre éstos dolores abdominales, diarrea y meteorismo post-prandial".

Si bien la ascaridiosis es generalmente benigna, puede provocar complicaciones de orden quirúrgico, generalmente de carácter obstructivo; estos accidentes se encuentran profusamente descritos en la literatura. Wagensteen ${ }^{7}$ indica como causa frecuente de obstrucciones intestinales en niños entre los 2 a 9 años, la presencia de paquetes de áscaris. Swart welder ${ }^{4}$, en un análisis de 202 casos de ascaridiosis, encuentra 18 obstrucciones intestinales, de las cuales 6 fueron mortales. Entre nosotros, Artigas, estudiando 89 enfermos con invaginación intestinal, eneuentra 1 caso debido a un paquete de áscaris. Einhorn ${ }^{\text {t }}$ comprueba que en 125 casos de infestación por áscaris en niños, sólo 2 pacientes presentaron trastornos agudos y ambos fallecieron, uno por peritonitis y hepatitis supurada, encontrándose áscaris en el antro mastoídeo. Ochner informa que los abscesos hepáticos debidos a los áscaris son generalmente múltiples y con pus fétido, pudiéndose abrir a la cavidad peritoneal y ocasionalmente al tórax. Entre nosotros, Meneghello y Pozo $^{R}$ descri- ben un caso de absceso hepático, en el cual se encontró un áscaris.

Nosotros hemos observado un paciente con ascaridiosis, que presentó diversas complicaciones de orden abdominal y que pasamos a relatar a continuación.

\section{E. A. R. - Obs. $51 / 90381$.}

Antecedentes inmediatos de ingreso: Se trata de un enfermo de 1 año 6 meses de edad, que presenteba desde hacía 2 meses episodios dispépticos alternados con breves períodos de bienestar; estos cuadros diarreicos se acompañaban de dolor abdominal y en algunas oportunidades se visualizaban áscaris en las deposiciones. El estado general del enfermo se fué progresivamente comprometiendo y un mes antes del ingreso apareció edema facial, aumento de volumen del abdomen, edema de las extremidades, tos, quejido y reacclón febril.

Antecedentes mórbidos familiares: Padre aparentemente sano. Madre sana. Se consigna un tio tuberculoso que convivió en el hogar durante los ültimos cuatro años.

Antecedentes rersonales: Nació el enfermo de un embarazo de término, siendo el parto y el período de recién nacido normales. Fué vacunado con B. C. G. en la maternidad. Alimentado al pecho durante las 3 primeros meses en forma desordenada. A continuación, por agalavtla se administraba en algunas ocasiones medio litro de leche de vaca mezclada con té o café; este último rógimen se suspendió porque el niño lo rechazaba. Al cumplir un año siguió el réglmen de la casa, que era deficiente. No se informa acerca de antecedentes mórbidos.

Examen físico de ingreso: Se comprueba un enfermo con marcado compromiso del estado general. sin preocupación por el medio ambiente, y que lloraba en forma monjtona. Existia un franco retraso pondoestatural $(7,600 \mathrm{~g} \mathrm{y} 70 \mathrm{~cm}$ de talia). Extensos signos policarenctales cutáneo mucosos: en el cuero 
cabelludo descamación fina y costras hemáticas. Hipertricosis fronto-parietal. Pestaña largas. Anemia Iranca de las encias. Lengua lisa rosarda pálida. Petequias en la cara anterior del cuello "y zonas discrómicas en la base. Petequias casi confluentes, formando equimosis en la región infraesternal y flanco izquierdo. Edema de la pared alodominal con ombligo evertido. Costras hemáticas en la pared anterior del tórax.

Extremidades superfores: edema franco de las manos y antebrazos; especialmente en la cara externa de las extremidades superiores se evidencla descamación furfurácea. melanosis y gratage.

Extremidades inferiores: edema franco de pies y piernas, más disereto a nivel de los muslos; enfriamiento y cianosis de los pies; extensa zona equimótica en la cara ánteroInterna del musio izquierdo; en la región supramaleoler interna derecha existe una zona simllar, pero más pequeña. Víblees en la articulación tibiotarsiana derecha $\mathrm{y}$ on la cara anterlor del muslo derecho. Descamación y melanodermia en la cara interna de los muslos. Descamación y erosión escrotal. Lesiones erosivas perianales.

Cangllos: microadenia inguinal bilateral.

Facie: pálida amarillenta. Abotagada. Cejas muy bien delineadas.

Abdomen: globuloso, con especial prominencia de los flancos. Submatidez en los flancas. Edema de la pared con prominencla del ombligo.

Hígado: hepatomegalia de $3 \mathrm{~cm}$ y de consistencia dura.

El resto del examen era negativo.

Impresión diagnóstica: De acuerdo con los antecedentes del enfermo y el examen físico, los dlagnósticos se orientaron en el sentido de una distrofia pluricarencial con edemas, sindrome purpúrlco y ascaridiasis.

Tratamientos: Ingresado al servicio se indicó alimentaclón a base de leche semidescremada, plasma y antibióticos. Durante el curso de la evolución se administró aureomicina. La ascaridiasis se trató mediante la santonina en cantidad de $0.0 \mathrm{t} \mathrm{g}$ por kilo de peso en 3 dosis, administrando al final $20 \mathrm{~g}$ de sulfato de magnesia.

Evolución: Llamó la atención el compromiso progresivo del estado general, la intensificación del edema, que posteriormente se localizó en la mitad ínferior del cuerpo; el aumento de la circunferencia abdominal con intensa infiltración edematosa de la pared acompañada de marcada circulación venasa colateral y signos clínicas de ascitis, siendo la palpación abdominal dolorosa. La ascaridiosis se confírmó, además, en la evoluclón, ya que el enfermo expulsó alrededor de 200 áscaris. Dradas las caracteristicas yo expuestas del cuadro abdominal, se pensó en la posibilidad de una peritonitis y con este objeto se practicaron paracentesis, dando la primera de ellas salida a abundante cantidad de líquido verdoso de aspecto purulento y las posteriores a escasas gotas de pus. El resultado de la primera punción reveló la existencla de abundantes glóbulos de pus, escasas bacterias de naturaleza dfversa (cocos y bacilos), la tánción de Zlehl fué negativa para el bacilo de Koch y el cultivo dió escaso desarrollo de colonias diferentes. Cada vez que se efectuaron estas punciones se inyectaron intraperitonealmente estreptomicina 1 g y 1 millón de unidades de penicilina. Frente a este cuadro peritoneal se llamó en intenconsulta al cirujano, quien pensó se tratara de una reritonitis "médica", secundaria a un cuadro séptico y aconsejó el tratamiento a base de punciones evacuadoras y antibióticas.

Tal como anteriormente se dijo, el estado general se fué progresivamente empeorando, aparecieron febriculas y crépitos en la base pulmonar derecha y submacidez, revelando la radioscopía de tórax la existencia de un pequeño derrame basal derecho. El abdomen aumentó tanto de volumen que el ombligo se evertló totalmente, apareciendo en su vêrtice una zona de piel delgada y amarillenta con la base infiltrada; fué en ese momento en que se decidió intervenir de urgencia, pero el enfermo falleció momentos antes.

Exámenes de laboratorio; Hemograma: G. R., 3.210,000; Hb., $36 \%$; V. G1., 0.56; reticulocitos, $6.3 \%$; micromacrocitosis de franco predominio microcitico; leucocitosis, 45,400; eos., 0; bas., 1; miel., 2; juv., 1 ; bac., 3; seg., 69; 7ln., 23; mon., 1. Neutrófilos con escasas granulaciones patológicas. Plaquetas normales. Indice ictérico normal. El mielograma indicaba signos de intensa reacción medular. En suma, existía anemia intensa con grandes alteraciones morfológicas (anisocitosis) y con graves deficiencias en la formación de la hemoglobina, signos de carácter regenerativos (reticulocitosis, policromatofilia), sin slgnos de hemolisis.

Pruebss de floculación: Hanger, + ; rojo escarlata, 0; timol turbidez, $15.8 \mathrm{U}$.

Proteinemias: 4.1 y $5.5 \mathrm{~g} \%$

La punción biopsia del hígado reveló un higado de primera categoría.

Fl electrocardiograma informó la existencia de daño miccárdico.

El enfermo fué enviado a la autopsia con los diagnósticos de síndrome policarencial con edemas, síndrome purpúrico, ascaridiasls $y$ peritonitis médica.

Informe anátomo patológico (necropsia $\mathrm{N}^{\mathrm{H}}$ 370): Anglocolitis supurada. Formación de abscesos colangiticos múltiples, algunos ablertos a la cavidad abdominal y otros al pericardio. Intensa colangiosis. Numerosos áscaris se encuentran en las vías blliares intra heráticas y en los abscesos hepáticos. Peritonitis fibrinopurulenta hemorrágica genera- 
lizada. Pericarditis fibrino purulenta. Tumefacción turbia del mfocardio, higado y riñones. Caquexia.

Hematoma intraparietal de un asa del yeyuno. Edema acentuado de los miembros inferiores. Focos de atelectasla de las zonas paravertebrales y cara externa de las lóbulos pulmonares. Edema del cerebro. Hernia um. bilical.

Exámenes histopatológicos:

Hígado: numerosos abscesas ocupados por tejido necrosado y abundante bilis rodeados For barrera leucocitaria. Parénquima con acentuada tumefacción turbia. Espacios portobiliares infiltrados discretamente por mononucleados.

Bazo: hiperplasia de las pulpas roja y blanca. La pulpa roja aparece con senos pequeños, limitados por endotelio cúbico redondeado. No se observa esplenitis aguda.

Exămenes ibacteriológicos de fibrina del peritoneo (Dr. Joanon):

Examen microsé́pico: (-).

Cultivo: hay desarrollo de B. Piociánico.

Pus de abscesos hepáticos.

Examen mícroscópico: (-).

Cultivo: hay desarrollo de B. Coli.

\section{Comentario.}

En el ingreso el enfermo impresionó como un distrófico pluricarencial con intenso edema y distensión abdominal, que se acompañaba de edema de la pared; sin embargo, posteriormente por el aumento considerable de la circunferencia abdominal, el compromiso progresivo del estado general, el dolor provocado por la palpación del abdomen, la presencia de circulación venosa colateral, unidas a una macidez desplazable, se planteó el diagnóstico de una peritonitis, la cual se comprobó al extraer de la cavidad peritoneal pus, que al examen mícroscópico reveló la existencia de cocos y bacilos diversos. Por la edad del enfermo y la evolución del cuadro clínico, pensamos que estábamos en presencia de una peritonitis de tipo médico secundaria a un cuadro de sepsis, cuyo punto de partida Ignorábamos.

Por estos motivos se indicó un tratamiento enérgico a base de antibióticos por vías general y local, aprovechando las punciones abdominales evacuadoras del pus peritoneal. Pese a este tratamiento, el estado general del paciente se fué comprometienđo cada vez más, por lo que hacia el día 27 se indicó intervención quirúrgica, que no se llevó a efecto por la muerte del enfermo.

La anatomía patológica comprobó la existencia de peritonitis $y$ pericarditis purulenta, lesiones de toxemia, angiocolitis supurada y abscesos colangíticos múitiples abiertos al abdomen y pericardio. La presencia de áscaris en las vias biliares y de abscesos hace pensar que estos parásitos, que se expulsaron en cantidad de más de 200 , han sido los agentes que condicionaron la infección de las vías biliares, punto de partida de la peritonitis del enfermo.

Las peritonitis en el niño menor, como también en otras edades, generalmente son secundarias a complicaciones de cuadros abdominales, las más de las veces agudos, que exigen un tratamiento quirúrgico, tales como la invaginación intestinal y la apendicitis ${ }^{8}$. Existen también en la menor edad peritonitis que podrian llamarse primarias, en las que la infección alcanza el peritoneo por vía hematógena, en el curso de procesos septicémicos ${ }^{k}$. La evolución clíníca de la observación relatada parecía corresponder a un cuadro peritoneal de este último tipo, y en este sentido se orientó el tratamiento, realizado a base de la administración de antibióticos asociados, inyectados por vías paraenteral y local.

La anatomía patológica señaló como causa del cuadro peritoneal la ruptura de abscesos hepáticos, no sosp̄echados en la clínica y en cuya génesis ha intervenido la ascaridiosis intrahepática que se comprobara.

\section{Sumario.}

Se relata la observación de un lactante de 1 año 6 meses que presentó peritonitis y pericarditis purulenta, como consecuencia de la ruptura de abscesos hepáticos múltiples. La existencia de numerosos áscaris en las vías biliares intrahepáticas $\mathrm{y}$ de angiocolitis supurada, permite suponer que estos parásitos han condicionado los abscesos del hígado y secundariamente el cuadro de peritonitis. 


\section{Summary.}

The author report a case of an 18 months old infant who had purulent pericarditis and peritonitis secondary to the rupture of multiple liver abscesses. The presence of many ascaris in the intrahepatic bile ducts and the suppurative angiocolitis, allows one to suspect that these parasites have conditioned the liver abscesses and secondarily the peritonitis.

\section{Bibliografía.}

1.-NIEGHME, A.; y SILVA, R. - Nueva contribuclón al estudio epidemiológico de la amebiasis $y$ otras entoparasitosis en thile. Rev. Méd, Ch. 79: 449, 1851.

2. WAGENGTFEN , B. A. - Intestinal Obstructions. Charies C. Thomas, springfield Illinois, 1942.
3.-CRAIG, C. F. and FAUST, E. C. - Clinical Parasitology. Lea-Febiger, Philadelphia, 1839.

4.-SWARTWETDER, - Clinical Ascaridiosis. Am. J. Dis. Child. 72: 172, 1046.

5.-ARTIGAS, $R$. - Invaginación Intestinal en el niño. Rev. Chjlena de Pediatría 60: 1051 .

6. -EINHORN, N. ; MILIER, J. F. and WHITTIER, I. - Clinical Survey of one hundred and twenty five cases of infection with Ascaris Lumbricoides in children. J. Tis. Child. 69: 237, 1945.

7. -OCHSNER, A.; DE BAKER, E. G. and DIXON, $\mathrm{L}$. - Complication of Ascarls requering surgical treatment. Report of case with abdominothoracic complication. Amer. J. Dis. of Chil, 77: 389, III-1949.

8.-MEENEGHELLO, J. y ROZO, s. - LOS abscesos hepáticos en la infancia. Rev. Chilena de Pediatria 9: 736, 1944.

9.-LITCHFIFLD, H. R. and DFMIBO, L. H. - Therapeutics of Infancy and Childhood. Philadelphia. F. A. Davis Company, Publishers. 1942. 